\title{
Molecular insights into the structural and dynamical changes of calcium channel TRPV6 induced by its interaction with phosphatidylinositol 4,5-bisphosphate
}

\author{
Lingyun Wang ${ }^{1}$, Ruiqi Cai ${ }^{2}$, Xing-Zhen Chen $^{2}$, and Ji-Bin Peng ${ }^{1}$ \\ ${ }^{1}$ The University of Alabama at Birmingham Division of Nephrology \\ ${ }^{2}$ University of Alberta Faculty of Medicine \& Dentistry
}

January 31, 2022

\begin{abstract}
Transient receptor potential vanilloid subfamily member 6 (TRPV6) is an epithelial $\mathrm{Ca}^{2+}$ channel that regulates the initial step of the transcellular $\mathrm{Ca}^{2+}$ transport pathway. TRPV6 is expressed in the kidney, intestine, placenta, and other tissues, and the dysregulation of the channel is implicated in several human cancers. It has been reported that phosphatidylinositol 4,5-bisphosphate (PIP 2 ) activates TRPV6 and its close homologue TRPV5; however, the underlying molecular mechanism is less clear. Recently, a structure of rabbit TRPV5 in complex with dioctanoyl ( $\mathrm{diC}_{8}$ ) PIP ${ }_{2}$, a soluble form of PIP ${ }_{2}$, was determined by cryo-electron microscopy. Based on this structure, the structural model of human TRPV6 with PIP 2 was set up, and then molecular dynamics simulations were performed for TRPV6 with and without PIP 2 . Simulation results show that the positively charged residues responsible for TRPV5 binding of diC $8 \mathrm{PIP}_{2}$ are involved in the interactions between TRPV6 and PIP ${ }_{2}$. The binding of PIP 2 to TRPV6 increases the distance between the diagonally opposed residues D542 in the selectivity filter and that between the diagonally opposed M578 residues in the lower gate of TRPV6. A secondary structural analysis reveals that residues of M578 in TRPV6 undergo structural and position changes during binding of PIP 2 with TRPV6. In addition, principal component analysis indicates that the binding of PIP 2 increases the dynamical motions of both the selectivity filter and the lower gate of TRPV6. These changes induced by PIP ${ }_{2}$ favor the channel opening. Thus, this study provides a basis for understanding the mechanism underlying the PIP ${ }_{2}$-induced TRPV6 channel activation.
\end{abstract}

\section{Hosted file}

proteins V6pip2-main.doc available at https://authorea.com/users/458233/articles/554818molecular-insights-into-the-structural-and-dynamical-changes-of-calcium-channel-trpv6induced-by-its-interaction-with-phosphatidylinositol-4-5-bisphosphate 